Pickett, M. J. \& Cabelli, V. J. (1953). J. gen. Microbiol. 9, 249-256.

\title{
The Precipitating Antigens of Friedländer's Bacillus
}

\author{
By M. J. PICKETT and V. J. CABELLI* \\ From the Department of Bacteriology, University of California, \\ Los Angeles, California
}

\begin{abstract}
SUMMARY: The biochemical characteristics of approximately eighty strains of Friedländer's bacillus are described. Certain aspects of their antigenic patterns were studied by testing the precipitating antigens of phase variants of originally mucoid strains, as well as the parent strains themselves. Evidence is presented that an as yet undescribed antigenic layer exists between those characteristic of the mucoid and smooth phases. This layer is most pronounced in the Sm phase organisms. The Sm antigen was shown to be present in nearly all Friedländer's strains and absent in the Bacterium coli strains studied; hence an Sm antiserum should be valuable for diagnostic purposes. The biochemical and serological results are discussed in regard to the taxonomy of this group with particular reference to the consolidation, already proposed by other workers, of the genera Klebsiella and Aerobacter.
\end{abstract}

In the studies to be described the precipitin patterns of phase variants of originally mucoid organisms were used to demonstrate another antigenic layer in addition to those described by Kauffmann (1949a) and to show the possibility of its use in the identification of members of the Klebsiella group. Edwards (1929), Julianelle (1930), Osterman \& Rettger (1941 $b$ ), and Kauffmann $(1949 a)$ studied the biochemical characteristics of the group (for earlier work see Topley and Wilson's Principles of Bacteriology, 1946). Their findings indicate an extreme variability in the biochemical reactions. Kauffmann (1949a) listed some general biochemical characteristics of the group. He defined the Klebsiella group as 'non-motile, Gram-negative, non-sporing rods that do not form indole, and which ferment adonitol, inositol and other carbohydrates or alcohols, often breaking down urea and frequently giving a positive VogesProskauer (VP) reaction and negative methyl-red (MR) reaction'. He went on to mention that the variable characteristics of the entire family Enterobacteriaceae make it necessary that all the above characteristics, rather than any one of them, be used in classifying these organisms.

Julianelle $(1926 a, 1926 b)$ first demonstrated the antigenic specificity of the capsular material and classified the organisms on that basis. Kauffmann $(1949 a)$ reported fifteen different serological groups on the basis of the specificity of the capsular $K$ antigen, and three main types on the basis of an antigenic carbohydrate underlayer ( $O$ antigen). The close serological relationship of Klebsiella pneumoniae to Aerobacter aerogenes has been demonstrated by Osterman \& Rettger $(1941 a, b)$ and by Kauffmann (1949a). Shared $\boldsymbol{O}$ antigens have been demonstrated between $\boldsymbol{K}$. pneumoniae and Bact. coli by

* Present address: Department of Bacteriology and Preventive Medicine, School of Medicine, University of Missouri, Columbia, Missouri. 
Kauffmann (1949a). Henriksen (1949), who used the complement fixation test, found cross reactions between mucoid strains of $\boldsymbol{K}$. pneumoniae and Bact. coli; however, no such reactions were obtained when he used the agglutination test.

\section{MATERIALS AND METHODS}

The organisms which were used in these studies consisted of stock strains of $K$. pneumoniae from our collection and of strains recently isolated from sputa and urines. Some of the stocks were copies of those used by Julianelle and by Kauffman. The strains were as follows:

Type A :

\section{Isolated from blood or sputum}

SA1 Julianelle's Sc strain, American Type Culture Collection no. 4208.

SA2 Levy-Bruhl's Bonneral strain.

SA3 Julianelle's Murtens strain.

SA4, SA5, SA6, SA7.

Type B :

SB 1 From Dr Gordon Nichols.

SB2 From New England Deaconess Hospital.

SB3 Julianelle's ' $E$ ' strain, pneumonia in a guinea pig.

SB 4, SB 5.

Type C:

SC 1 Julianelle's F-10 strain.

SC2, SC3, SC4.

K11 Kauffmann's 5056 strain.

SX 1 Group ' $X$ '.

\section{Isolated from urine}

UA1 Type A.

UB1, UB2, UB3 Type B.

UC1 Type $C$.

UX1-36 Group ' $X$ '.

K1 Kauffmann's 1193 strain.

K2 Kauffmann's K 390 strain.
K3 Kauffmann's K56 strain.

K4 Kauffmann's 1015 strain.

K6 Kauffmann's 373 strain.

K 8 Kauffmann's 919 strain.

UI 1-10 Indole positive.

\section{Rhinoscleroma and Ozaenae strains}

K5 Kauffmann's Rhinoscleroma strain 5046-type C.

K9 Kauffmann's Ozaenae strain 5050-type D.

K10 Kauffmann's Ozaenae strain 5052-type F.

K12 Kauffmann's Ozaenae strain 5051-type E.

Cells from a single colony on a peptone agar plate were subcultured to peptone broth and then to the various test media. The MR and VP tests were read after $36 \mathrm{hr}$. The rest of the tests were read after $24 \mathrm{hr}$. and at 1 day intervals thereafter to the 15 th day.

The strains were characterized serologically by the use of the interphase precipitin test. Growth from an $18 \mathrm{hr}$. peptone agar slope culture was emulsified in approximately $10 \mathrm{ml}$. saline and centrifuged at 3000 r.p.m. until a clear 
supernatant was obtained. The supernatant containing the extracted material was layered by means of a capillary pipette over undiluted antisera in tubes whose inside diameters were about $3 \mathrm{~mm}$. The tubes were left at room temperature and read at 5 and $30 \mathrm{~min}$. intervals. This procedure was used for the mucoid parent strains as well as for the phase variants. In order to extract the Sm antigen from mucoid organisms (Table 3, labelled (heat)), the cell suspensions were heated at $100^{\circ}$ for $7 \mathrm{~min}$. prior to centrifugation. A positive test manifested itself as a precipitate at the interface. Positive and saline controls were performed with each run.

All the antisera used in this study were prepared by injecting rabbits with increasingly heavy $0.4 \%$ phenolized saline suspensions of unwashed cells grown on peptone agar slopes. The antigens were injected every other day for 3 months. Strains SA1, SB2 and SC1 were used to prepare anti-A, anti-B, and anti-C sera, respectively. Anti-Sm was a bivalent serum against strains UX35 and UX36. In the preparation of anti-Sm serum, however, washed cell suspensions were used. Unwashed and unheated cell suspensions of SB2s, a smooth variant of SB2 which had lost its ability to react with anti-B or anti-Sm sera but which had retained the $O$ antigenic layer, were used for the preparation of the anti-O serum.

The colonial and microscopic morphologies of the stock strains and the variants which were obtained in the course of repeated passage through brilliant green were studied, and their antigenic precipitin patterns were determined by using the sera mentioned above. In order to obtain the variants, the types $\mathrm{A}, \mathrm{B}$, and $\mathrm{C}$ parent strains were inoculated in duplicate to $16 \mathrm{~mm}$. tubes containing $10 \mathrm{ml}$. of a medium composed of $1 \%$ peptone, $0.5 \% \mathrm{NaCl}$, and brilliant green to a concentration of $1: 10^{7}$ in distilled water; the tubes were then incubated at $37^{\circ}$. Subcultures were made every other day to the medium mentioned above; however, the concentration of the dye was increased when good growth was obtained in the previous subculture. A $0.1 \mathrm{ml}$. inoculum was generally used; but, when no growth was obtained in a higher concentration of the dye, the amount was increased up to $1.0 \mathrm{ml}$. At weekly intervals samples were taken and plated on peptone agar for the detection of the variants which were then picked off and replated to the same medium. They were so replated on at least four successive days at the end of which time they were streaked on peptone agar slopes and kept in stock for later use. At the end of 3 months, when a representative number of variants had been obtained and when all the parent strains were growing in a 1:50,000 concentration of brilliant green, the collected variants were replated on peptone agar from which representative colonies were restreaked to slopes for the precipitin tests.

\section{RESULTS}

The variability in most of the biochemical reactions can be seen from the results summarized in Table 1. Maltose, sucrose, galactose, mannitol, sorbitol, mannose, xylose and arabinose were almost always fermented in $24 \mathrm{hr}$. Most of the strains which were isolated from urine resembled UX1 and UX 7 both biochemically and serologically. 
We would like to point out that the lactose-negative strains are much less reactive biochemically than the lactose-positive ones. The lactose-negative strains have almost or completely lost the ability to produce acetylmethylcarbinol and to ferment dulcitol and inositol; it was also found that they were unable or only weakly able to use ammonia as a source of nitrogen.

Table 1. Biochemical characteristics of the strains of Friedländer's bacillus examined

$\begin{array}{lcccccc}\text { Strain } & \text { MR } & \text { VP } & \text { Ind } & \text { Inos } & \text { Dulc } & \text { Lact } \\ \text { SA1, 3 } & + & + & - & +10 d & - & - \\ \text { SA2, 5 } & - & + & - & + & + & + \\ \text { SA4, 6 } & + & - & - & +10 d & - & - \\ \text { SA7 } & - & + & - & + & - & + \\ \text { SB1 } & \pm & + & - & + & - & + \\ \text { SB2 } & \pm & + & - & +2 d & - & + \\ \text { SB3, 4 } & - & + & - & + & - & + \\ \text { SB5 } & - & + & - & + & - & + \\ \text { SC1, 2 } & - & + & - & + & + & + \\ \text { SC3 } & + & - & - & + & + & + \\ \text { SC4 } & - & + & - & +6 d & - & +2 d \\ \text { SX1 } & - & + & - & + & + & + \\ \text { UA1 } & - & + & - & + & + & + \\ \text { UB1, 2 } & - & + & - & + & - & + \\ \text { UB3 } & - & + & - & + & - & + \\ \text { UC1 } & \pm & \pm & - & + & + & + \\ \text { UX2 } & + & + & - & - & - & +3 d \\ \text { UX3 } & + & \pm & - & + & + & + \\ \text { UX4 } & + & + & - & + & + & + \\ \text { UX7 } & + & - & - & + & - & + \\ \text { UX14 } & + & + & - & + & + & + \\ \text { UX35 } & - & + & - & + & - & + \\ \text { UX36 } & + & - & - & +2 d & - & + \\ \text { K1, 2, 3 } & - & + & - & + & - & + \\ \text { K4,6 } & - & + & - & + & - & + \\ \text { K5 } & + & - & - & + & - & + \\ \text { K8 } & + & + & - & + & + & + \\ \text { K9 } & + & - & - & + & - & + \\ \text { K10 } & + & - & - & +2 d & - & + \\ \text { K11 } & + & - & - & +2 d & + & + \\ \text { K12 } & - & + & - & + & + & + \\ \text { K12 } & + & + & - & +\end{array}$

MR = Methyl red; VP=Voges-Proskauer ; Ind =Indole ; Inos = Inositol; Dulc=Dulcitol; Lact $=$ Lactose; $d=$ days; $+=$ posititive; $-=$ negative; $\pm=$ slightly positive.

The serological characteristics of the strains are presented in Table 2 . We would like to call special attention to the almost uniform reaction with the bivalent serum, anti-Sm. All the strains of Bact. coli which were tested were uniformly negative with the anti-Sm serum.

The colonial morphologies of the phase variants are to be seen in $\mathrm{Pl}$. 1, figs. 1-5. Microscopically, coccobacilli were the predominant forms in the mucoid phase. Rough phase variants were composed of numerous long rods, swollen forms, and long filaments, some of which extended the length of the visual field. Phase variants between these two extremes showed gradations in form consistent with the colonial morphology by which they were designated.

The precipitin pattern of the phase variants of strains SA1, SB2, SC2, 
UX35 and UX36 are shown in Table 3. The latter two strains are those used to prepare the bivalent serum. The results point to the existence of at least three distinct soluble antigenic layers, in addition to those possessed by the rough phase organisms.

Table 2. Serological characteristics of the strains of Friedländer's bacillus examined

\begin{tabular}{lllll} 
& \multicolumn{4}{c}{ Antisera } \\
\cline { 2 - 5 } Strain & A & B & C & Sm \\
SA1, 3 & + & - & - & + \\
SA2,5 & + & - & - & + \\
SA4,6 & + & - & - & + \\
SA7 & + & - & - & + \\
SB1 & - & + & - & + \\
SB2 & - & + & - & + \\
SB3, 4 & - & + & - & + \\
SB5 & - & + & - & + \\
SC1, 2 & - & - & + & + \\
SC3 & - & - & + & + \\
SC4 & - & - & + & + \\
SX1 & - & - & - & + \\
UA1 & + & - & - & + \\
UB1, 2 & - & + & - & + \\
UB3 & - & + & - & - \\
UC1 & - & - & + & + \\
UX2 & - & - & - & + \\
UX3 & - & - & - & + \\
UX4 & - & - & - & + \\
UX7 & - & - & - & + \\
UX14 & - & - & - & + \\
UX35 & - & - & - & + \\
UX36 & - & - & - & + \\
K1, 2, 3 & - & - & - & + \\
K4,6 & - & - & - & + \\
K5 & - & - & - & + \\
K8 & - & - & - & + \\
K9 & - & - & - & + \\
K10 & - & - & - & + \\
K11 & - & - & + & + \\
K12 & - & - & - & + \\
S12 & & - & +
\end{tabular}

$-=$ no precipitate using the raw antiserum.

$+=$ the formation of a precipitate at the interphase.

\section{DISCUSSION}

The results leave no doubt that biochemical tests do not offer a very satisfactory basis for the differentiation of the genus Klebsiella from the genus Bacterium (Escherichia). Of them all, the indole reaction and the fermentation of inositol as exclusion tests seem the most reliable. The authors feel, as did Kauffmann (1949 $a, 1949 b)$ that the genera Klebsiella and Aerobacter should be fused into a single genus since they can be differentiated neither biochemically nor serologically.

The serological identification on the basis of capsular antigens has been quite adequately covered by Kauffmann (1949a); the large number of types 
Table 3. Precipitin reactions of phase variants

\begin{tabular}{|c|c|c|c|c|c|c|}
\hline \multirow[b]{2}{*}{ Strain } & \multirow[b]{2}{*}{ Phase } & \multicolumn{5}{|c|}{ Antisera } \\
\hline & & $\mathbf{A}$ & $\mathbf{B}$ & C & Sm & O \\
\hline SA 1 & m & $++t$ & . & . & - & - \\
\hline SA 1 & ms & + & . & . & $+t+$ & +++ \\
\hline SA 1 & sm & + & . & - & ++ & $+t+t$ \\
\hline SA 1 & $\mathbf{s}$ & \pm & - & . & - & $+t+$ \\
\hline SA 1 & $\mathbf{r}$ & - & . & - & - & - \\
\hline SB2 & $\mathbf{m}$ & . & ++++ & . & + & - \\
\hline SB2 & m (heat) & . & $+t+t$ & . & $++t$ & - \\
\hline SB2 & sm & . & ++ & . & $+t+$ & +++ \\
\hline SB2 & $\mathbf{s}$ & . & - & . & - & ++ \\
\hline SB2 & $\mathbf{r}$ & . & - & . & - & $-\bullet$ \\
\hline SB3 & m & . & ++++ & . & - & - \\
\hline SB3 & m (heat) & . & $++t+$ & . & +++ & - \\
\hline SB3 & sm & . & ++ & - & ++++ & +++ \\
\hline SB3 & $\mathbf{s}$ & - & - & - & - & +++ \\
\hline SB3 & $\mathbf{s r}$ & . & - & • & - & ++ \\
\hline SC2 & $\mathbf{m}$ & . & . & $+t+t$ & - & - \\
\hline $\mathrm{SC2}$ & ms & . & . & +++ & $+t+$ & ++ \\
\hline SC2 & $\mathbf{s}$ & . & . & - & - & +++ \\
\hline SC2 & rs & . & . & - & - & + \\
\hline UX35 & m & - & - & - & $+t+$ & - \\
\hline UX35 & m (heat) & . & . & . & $+t+$ & ++ \\
\hline UX36 & m & . & - & - & $+t+$ & - \\
\hline UX36 & m (heat) & . & . & . & $++t$ & - \\
\hline UX36 & ms & . & - & . & $++t$ & - \\
\hline UX36 & $\mathbf{s}$ & . & . & - & - & ++ \\
\hline UX36 & $\mathbf{s r}$ & . & - & - & - & ++ \\
\hline UX36 & $\mathbf{r}$ & . & . & . & - & - \\
\hline
\end{tabular}

Heat $=$ suspension heated at $100^{\circ}$ for 7 min. prior to centrifugation.

++++ to $-=$ strong to negative precipitate at the interphase.

$\mathrm{m}=$ mucoid ; $\mathrm{ms}=$ mucoid-smooth; $\mathrm{sm}=$ smooth-mucoid; $\mathrm{s}=$ smooth ; $\mathrm{sr}=$ smooth-rough; rs = rough-smooth; $r=$ rough.

$\mathrm{O}=$ agglutination with appropriate $\mathrm{O}$ sera.

and the discovery of new ones (Miss Kelly, personal communication) makes identification by this means impractical. It was to this end that the bivalent serum was used in the hope that it could be utilized as an easy means of identification and classification. To be of value the serum would have to react with all mucoid and smooth-mucoid strains of $K$. pneumoniae and give negative precipitin tests with all other species. It is felt that the first criterion has been satisfied, but more extensive tests with related organisms, i.e. Bacterium (Escherichia) spp., Proteus spp., etc., are necessary before a final statement on the second point can be made.

Although absorption tests would be needed definitely to prove the identity of the precipitating antigens of $\boldsymbol{K}$. pneumoniae, certain conclusions can be drawn as to their nature as seen from the data of Table 3:

1. The Sm antigen or antigens are distinct from and lie beneath the capsular $\mathrm{A}, \mathrm{B}$, and $\mathrm{C}$ antigens. This is shown by the fact that anti-Sm serum will not precipitate the supernatant from mucoid $\mathrm{A}, \mathrm{B}$ or $\mathrm{C}$ strains, but will precipitate the supernatant from all three if the cell suspensions are first heated. 
2. The $\mathrm{Sm}$ antigen or antigens are distinct from the $\mathrm{O}$ antigens. This is borne out by the fact that $\mathrm{Sm}$ antiserum but not $\mathrm{O}$ will react with the supernatant from heated suspensions of mucoid phase organisms, while the $\mathbf{O}$ and not the Sm antiserum will react with that from smooth phase cells.

3. In view of the preceding it can be said that there are at least three distinct antigenic layers exclusive of the one characteristic of the rough phase.

In view of the peculiarities of the Sm antigen, one can see why it could have been overlooked by previous workers. Since it is absent in the smooth organisms, anti-smooth sera would not contain antibodies against it. Antibodies against it may be present in anti-mucoid sera, but the suspensions would have to be heated before testing the supernatant in order to obtain a positive precipitin reaction. Conceivably, antibodies against the Sm antigen could be absent from anti-(m)sera if the cell suspension is neither heated nor washed before injection, if it is used as soon as prepared, or if the immunization time is short.

Finally, the ability to detect the Sm antigen was dependent upon the fortuitous finding of strains UX35 and UX36 in which the Sm antigen was readily exposed by washing alone (Table 3). Hence, anti-Sm antibodies were produced when only washed cell suspensions were used as the immunizing antigen.

\section{REFERENCES}

Edwards, P. R. (1929). Relationships of the encapsulated bacilli with special reference to Bact. aerogenes. J. Bact. 17, 339.

Henriksen, S. D. (1949). Sero-types of a collection of mucoid Gram-negative rods. Acta path. microbiol. scand. 26, 424.

Julranelue, L. A. (1926a). A biological classification of Encapsulatus pneumoniae (Friedländer's bacillus). J. exp. Med. 44, 113.

Julianelle, L. A. (1926b). Immunological relationships of encapsulated and capsule-free strains of Encapsulatus pneumoniae (Friedländer's bacillus). J. exp. Med. 44, 683.

Julianelue, L. A. (1930). The distribution of Friedländer's bacilli of different types. J. exp. Med. 52, 539.

KaUfFManN, F. (1949a). On the serology of the Klebsiella group. Acta path. microbiol. scand. 26, 381.

Kauffmann, F. (1949b). On the classification of the Enterobacteriaceae. Acta path. microbiol. scand. $26,879$.

Osterman, E. \& Rettger, L. F. (1941 $a$ ). A comparative study of organisms of the Friedländer and coli-aerogenes groups. I. Morphological and cultural characteristics, with emphasis on variation. $J$. Bact. 42,699 .

Osterman, E. \& Rettger, L. F. (1941 b). A comparative study of organisms of the Friedländer and coli-aerogenes groups. II. Pathogenicity, biochemical reactions, and serological relationships. J. Bact. 42, 721.

Topley and Wilson's Principles of Bacteriology and Immunology (1946). 3rd ed. Revised by Wilson, G. S. \& Mress, A. A. Baltimore: Williams \& Wilkins Co. 


\section{EXPLANATION OF PLATE}

Colonial characteristics of phase variants of Klebsiella pneumoniae strain SA1 on peptone agar. $\times 7$.

Fig. 1. Mucoid colonies.

Fig. 2. Smooth-mucoid colony.

Fig. 3. Smooth colonies.

Fig. 4. Smooth-rough and rough-smooth colonies.

Fig. 5. Rough colonies.

(Received 23 March 1953) 
Journal of General Microbiology, Vol. 9. No. 2
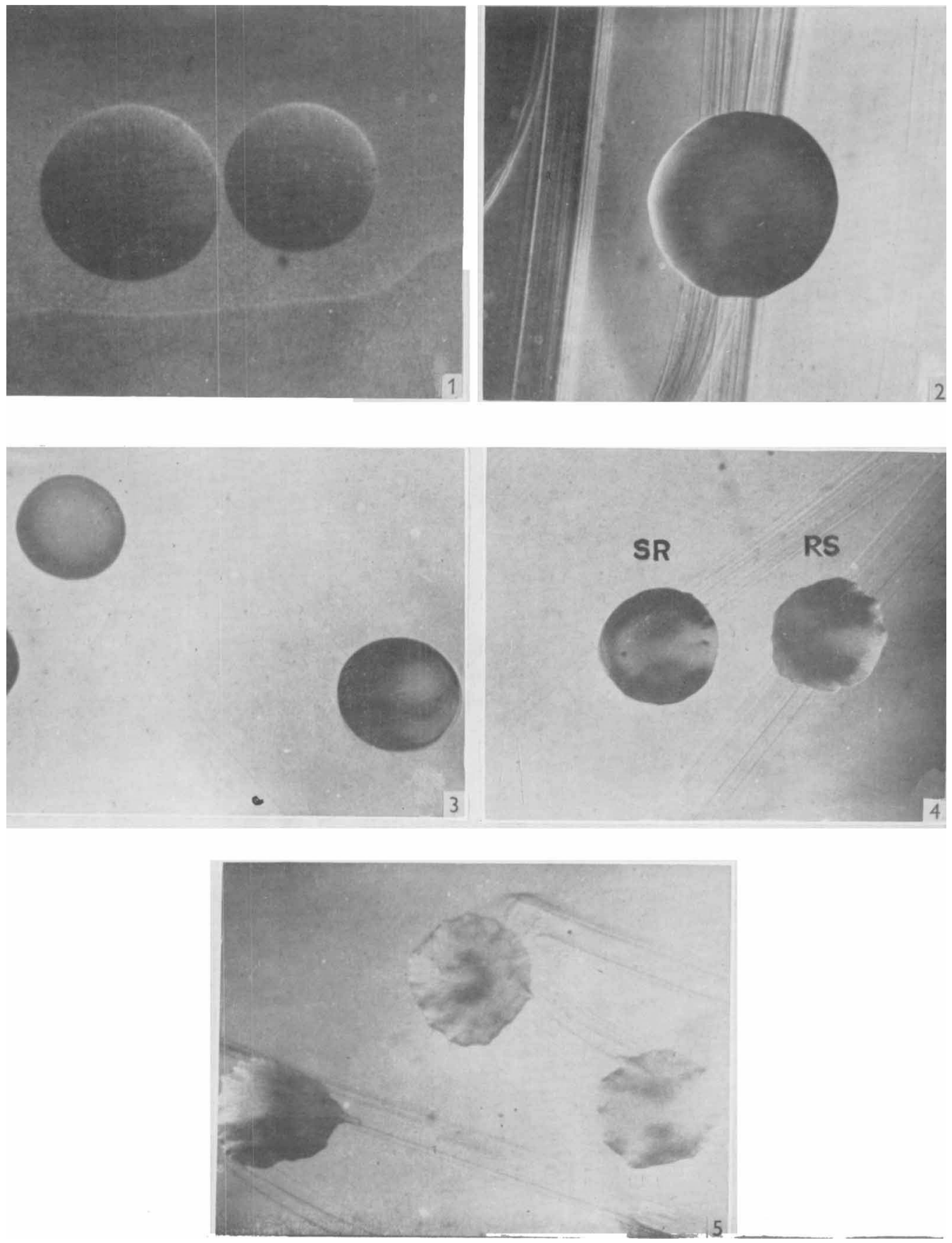

M. J. Pickett \& V. J. Cabelli-Antighens of Frindländer's bacillue. Plate 1 